\title{
Article
}

\section{The Relationship between Eccentric Hamstring Strength and Dynamic Stability in Elite Academy Footballers}

Rhodes, David, Jeffrey, Josh, Maden-Wilkinson, Joe, Reedy, Antony, Morehead, Erin Kristen, Kiely, John, Birdsall, Daniel, Carling, Christopher and Alexander, Jill

Available at http://clok.uclan.ac.uk/33655/

Rhodes, David ORCID: 0000-0002-4224-1959, Jeffrey, Josh, Maden-Wilkinson, Joe, Reedy, Antony, Morehead, Erin Kristen ORCID: 0000-0002-4096-0220, Kiely, John ORCID: 0000-0001-9817-0224, Birdsall, Daniel, Carling, Christopher ORCID: 0000-0002-7456-3493 and Alexander, Jill ORCID: 0000-0002-64921621 (2021) The Relationship between Eccentric Hamstring Strength and Dynamic Stability in Elite Academy Footballers. Science and Medicine in Football, 5 (1). pp. 48-54. ISSN 2473-3938

It is advisable to refer to the publisher's version if you intend to cite from the work. http://dx.doi.org/10.1080/24733938.2020.1782458

For more information about UCLan's research in this area go to http://www.uclan.ac.uk/researchgroups/ and search for <name of research Group>.

For information about Research generally at UCLan please go to http://www.uclan.ac.uk/research/

All outputs in CLoK are protected by Intellectual Property Rights law, including Copyright law. Copyright, IPR and Moral Rights for the works on this site are retained by the individual authors and/or other copyright owners. Terms and conditions for use of this material are defined in the policies page. 
2 The Relationship between Eccentric Hamstring Strength and Dynamic Stability in Elite Academy 3 Footballers.

4

5 Authors:

6 David Rhodes $^{\mathrm{a}}$., Josh Jeffrey ${ }^{\mathrm{b}}$., Joe Maden-Wilkinson ${ }^{\mathrm{b}}$., Antony Reedy ${ }^{\mathrm{c}}$., Erin Morehead ${ }^{\mathrm{a}}$. John Kiely ${ }^{\mathrm{a}}$, 7 Daniel Birdsall ${ }^{\mathrm{a}}$, Chris Carling ${ }^{\mathrm{a}}$, , and Jill Alexander ${ }^{\mathrm{a}}$.

8

$9 \quad$ a University of Central Lancashire, Preston, Lancashire, PR1 2HE, United Kingdom.

10 beverton FC, Finch Farm, Merseyside, United Kingdom.

11 'Burnley FC, Gawthorpe Park, Padiham, United Kingdom.

\section{Corresponding Author:}

Dr David Rhodes

DRhodes2@uclan.ac.uk

Word Count:

Declarations of interest:

None 


\section{ABSTRACT}

Objectives: Previous research describes dynamic stability and functional strength as key aetiological risk factors associated with lower limb non-contact musculoskeletal injury. Due to the multi factorial nature of injury risk, relationships between the two factors will inform injury management and training design.

Methods: Fifty-nine elite academy footballers from two English premier league category 1-status academies completed the study. All players completed measures of eccentric hamstring strength and dynamic stability. Relationships between directional stability (Anteroposterior (Ant), Posteromedial (PM) and Posterolateral (PL)) and eccentric strength metrics (PkT, AvT, PkF, AvF and $\Theta$ ) bilaterally were identified for analysis.

Results: Significant correlations were identified bilaterally for functional hamstring strength metrics and PM and PL stability $(\mathrm{P} \leq .0 .05)$. No significant relationships were identified between anterior stability and eccentric hamstring strength parameters $(\mathrm{P}>0.05)$.

Conclusions: Eccentric hamstring strength has a positive influence on directional stability through two planes, PM and PL. The lack of influence of eccentric hamstring strength on Ant directional stability could be attributed to increased ACL risk. Careful consideration of the significance of the relationships between eccentric hamstring strength and directional stability must be given when quantifying injury risk in elite academy footballers.

Keywords: soccer, eccentric, dynamic stability, injury 
57

Epidemiological research within elite football highlights the incidence of muscular injury, citing that they contributed to $31 \%$ of all injuries with injury most commonly occurring to the hamstrings ${ }^{1}$. These injuries alone in the English Premier League cost clubs in the region of $£ 74.4$ million in a single season and $£ 1$ billion across the whole of English Football ${ }^{2}$. Indications in the research literature illustrate that hamstring injury incidence has increased ${ }^{1,3}$, thus increasing the financial burden on placed on professional football clubs. Coincidentally, research on the continent has reported rises in anterior cruciate ligament injuries (ACL) ${ }^{4,5}$. In addition literature also highlights the detrimental effect of ACL rupture on subsequent levels of performance ${ }^{6}$ and the increased chance of re-rupture, with reductions of eccentric hamstring strength highlighted as a key factor?

Considering basic functional anatomy and the common mechanism of injury associated with ACL injury in footballers, it seems logical to suggest that the functional strength of the hamstring would be a key factor in maintaining stability within the knee, particularly through the anterior plane. Evidence documents that athletes partaking in injury prevention programmes focussing on improving functional hamstring strength parameters are at a reduced risk of sustaining knee injury ${ }^{8}$. Aetiological risk factors associated with sustaining knee injuries include poor eccentric strength ${ }^{9,10}$ and dynamic stability ${ }^{11}$. The work of Booysen et al., (2015) ${ }^{12}$ examined the relationship between these two risk factors concluding that no correlation exists. It is important to note that within this work dynamic stability was quantified utilising an overall stability score when performing the ' $\mathrm{Y}$ ' Balance Test (YBT). Consideration must be given to the importance of establishing the relationship between functional strength of the hamstrings and directional dynamic stabilisation, as this will inform injury risk reduction strategies employed by sports medicine professionals. In functional performance the hamstrings play a vital role in counteracting the stresses on the ACL when competing ${ }^{13}$. Thus, suggesting that poor eccentric control may result in decreased directional dynamic stability within the knee, increasing the athlete's chance of sustaining injury. 
Although the consensus across literature appears clear for the hamstring, the effect of increased functional hamstring strength on the stability of the knee is limited. Research highlights that players who sustain an ACL rupture display significant reductions in hamstring function, post injury or surgical intervention $^{14,15}$, increasing the risk of re-rupture. Previous injury is highlighted as a key aetiological factor in the recurrence of ACL ruptures ${ }^{16,17}$. This potentially highlights that returning an athlete post injury with poor function compromises the stability of the knee and exposes the ACL to increased load ${ }^{18}$. It is clear that poor function within the hamstring muscle group can contribute to non-contact musculoskeletal injury sustained at the knee ${ }^{19}$. Aetiological contributions to sustaining such injuries are well documented as being multi factorial, particularly literature surrounding the $\mathrm{ACL}^{20}$. This said, the global effect functional strength has on these aetiological factors is not well reported and further research is required.

During functional performance, changes in muscle length or alterations in knee position initiate a stabilisation response, due to the stimulation of mechanoreceptors within the joint or the muscle ${ }^{21}$. Key receptors responsible for this afferent response include Muscle Spindles, Golgi Tendon Organs (GTO's), Ruffini Endings, Ruffini Corpuscles and Pacinian Corpuscles ${ }^{11,22}$. As to which receptors stimulate the afferent signal however, is arguably irrelevant, as proprioceptive responses from the mechanoreceptors within the joint and muscle are likely to occur at the same time. Reducing injury risk associated with the hamstrings and the knee is reliant on the muscle's ability to generate the required effected functional response ${ }^{9}$.

Research has focussed on eccentric hamstring strengthening interventions to decrease injury occurrence in the hamstrings and the knee ${ }^{23}$. This experimental paradigm fails to consider the effect of functional training on other aetiological factors associated with injury, such as dynamic stability. It also fails to contemplate the multi factorial nature of sustaining these injuries ${ }^{24}$. The aim of the current study is therefore to establish whether a relationship exists between eccentric strength and stability performance. Establishing this relationship would inform the design of conditioning protocols implemented to reduce injury risk in athletes. It was hypothesised that high eccentric strength measures would relate to low 
dynamic stability scores. In consideration of the specific nature of the evidence base in regards to injury epidemiology, a population of elite youth soccer players took part in the current study.

\section{Methods}

Fifty-nine elite academy male footballers from two English premier league category 1-status academies completed the present study, age $17.98 \pm 2.29$ years; height $180.40 \pm 7.93 \mathrm{~cm}$ and weight $73.65 \pm 6.38 \mathrm{~kg}$. All players were in full training, free from injury and available for competitive selection. Any player who had returned from injury within two months leading to the study were not included. All participants provided written informed consent in accordance with department and faculty research ethics committees (STEMH), and in accordance with the Helsinki Declaration.

Participants completed a familiarisation trial 7 days prior to testing to negate potential learning effects ${ }^{25}$, which included 3 repetitions of the Nordic Hamstring Curl on the NordBord and the YBT. Prior to any testing all participants completed a standardised warm up (5 minutes cycling at submaximal intensity, a combination of skipping, high knees and butt kicking drills, 10 forward lunges per leg and 2 Nordic hamstring movements with low resistance ${ }^{26}$. All testing was completed between 13:00 and 17:00 hrs to account for the effect of circadian rhythm and in accordance with regular competition times ${ }^{27}$.

All strength testing was completed on the NordBord (Vald Performance) to determine eccentric knee flexor strength; with its reliability, being previously described ${ }^{24}$. Players knelt on a padded board, with the ankles secured immediately superior to the lateral malleolus by individual ankle braces that were attached to custom made uniaxial load cells (Delphi Force Measurement, Gold Coast, Australia) with wireless data acquisition capabilities (Mantracourt, Devon, UK). Post completion of the standardised warm up previously described participants completed 1 set of 3 maximal repetitions of the bilateral Nordic Hamstring exercises. Instructions to the players were to gradually lean forward at the slowest possible speed while maximally resisting this movement with both limbs while holding the trunk and hips in a neutral position throughout and the hands held across the chest. Participants were loudly 
exhorted to provide maximal effort throughout each repetition. A trial was deemed acceptable when the

140 force output reached a distinct peak (indicative of maximal eccentric strength), followed by a rapid

141

142 decline in force when the participant was no longer able to resist the effects of gravity acting on the segment above the knee joint ${ }^{26}$. In synchronisation with the eccentric strength parameters quantified via the Nordbord, joint kinematics were also measured during each repetition. Joint kinematics were recorded from the sagittal plane using a Canon XA35 camera (Figure 1). The camera was placed on a fixed stand set $3 \mathrm{~m}$ away and $0.5 \mathrm{~m}$ from the floor. Three reflective circular markers were attached to the right greater trochanter, right lateral femoral condyle, and right lateral malleolus to calculate knee joint kinematics. Minimal clothing was recommended to avoid movement of the markers. The Nordic hamstring exercise completed on the NordBord was analysed using a variation of the motion analysis protocol adopted from a previous study ${ }^{28}$. Video clips were digitized and transformed into a twodimensional space using motion analysis application software (IOS Nordics Application). Each participants' break point angle was calculated using the reflective markers placed on the landmarks previously set.

To ascertain dynamic stability measures for each athlete the YBT owing to its moderate-excellent reliability ${ }^{29}$ accompanied with its ease of use within a sporting environment. Participants were asked to remove their shoes and socks and completed testing on both lower limbs. This was to eliminate stability and balance provided by the footwear. Once stood single leg on the centre plate participants were asked to push the reach indicator block with the contralateral limb in the anterior (Ant), posteromedial (PM) and posterolateral (PL) directions. Each subject maintained a single leg stance with their hands on the pelvis whilst pushing the reach indicator as far as possible in each of the directions listed. A trial was classified as invalid if the participant did not return to the start position, failed to maintain a unipodal stance on the platform, kicked the reach indicator block to gain more distance, stepped on top of the reach indicator for support or removed their hands from their hips. 


\section{Data Analysis}

168

Participants completed three repetitions on the YBT and the NordBord. YBT scores in each direction

(Ant, PM and PL) were normalised for lower limb length to calculate maximum distance (\%MAXD)

171 utilising the following equation excursion distance/limb length $\mathrm{x} 100=\%$ MAXD. This accounted for potential differences in leg lengths amongst individuals ${ }^{30}$. Mean distance in each direction on the right and left sides were utilised for data analysis. Eccentric strength data for both left and right limbs is expressed as Newtons (N), Force (F), Peak Torque (PkT) and Average Torque (AvT) were identified for each participant and utilised for analysis. In addition, break angle $(\Theta)$ for each of the repetitions completed was taken and the mean $(\Theta)$ used for analysis.

177

\section{Statistical Analysis}

179

Pearson's correlation coefficients were calculated to quantify the linear relationship between YBT and eccentric strength profiles. All statistical analysis was completed using PASW Statistics Editor 25.0 for windows (SPSS Inc, Chicago, USA). Statistical significance was set at $P \leq 0.05$. Coefficient of correlation ( $\mathrm{r}$ ) and respective level of significance ( $\mathrm{p}$ value) describes total variance. The following criteria quantified magnitude of the correlation $<0.1$, trivial; $>0.1$ to 0.3 , small; $>0.3$ to 0.5 , moderate; $>0.5$ to 0.7 , large; $>0.7$ to 0.9 , very large; and $>0$ to 1.0 , almost perfect.

\section{Results}

Table 1 summarises the mean and standard deviation scores achieved for all metrics observed within the present study. 
Correlations displayed between directional dynamic stability scores quantified with the YBT and eccentric hamstring strength metrics ascertained through NordBord testing demonstrated statistically significant correlation coefficients for all eccentric hamstring strength metrics against right (PkT (L): $P$ $=0.04, r=0.37 ; \operatorname{PkT}(\mathrm{R}): P=0.04, \mathrm{r}=0.37 ; \operatorname{AvF}(\mathrm{L}): P=0.03, r=0.32 ; \operatorname{AvF}(\mathrm{R}): P=0.003, r=$ $0.39 ; \operatorname{AvT}(\mathrm{L}): P=0.005, r=0.36 ; \operatorname{AvT}(\mathrm{R}) P=0.04, r=0.34)$ and left posterior-medial stability (PkT (L): $P \leq 0.001, r=0.60 ; \operatorname{PkT}(\mathrm{R}): P \leq 0.001, r=0.59 ; \operatorname{AvF}(\mathrm{L}): P=0.01, r=0.32 ; \operatorname{AvF}(\mathrm{R}): P=0.03$, $r=0.31 ; P \leq 0.001, r=0.62 ; \operatorname{AvT}(\mathrm{R}) P=0.03, r=0.38)$. No significant correlation coefficients were found between break angle and right and left posterior-medial stability $((\mathrm{R}) \mathrm{PM}: P=0.59, r=-0.07$; (L) PM: $P=0.58, r=0.07$ ). The magnitude of the statistically significant correlations ranging from small to moderate.

Right limb posterolateral stability displayed significant correlation coefficients against PkT (R) and (L) $(\operatorname{PkT}(\mathrm{R}): P \leq 0.001, r=0.42 ; \mathrm{PkT}(\mathrm{L}): P \leq 0.001, r=0.41) ; \operatorname{AvF}(\mathrm{R})(P=0.05, r=0.32)$ and AvT (R) $(P=0.002, r=0.40)$. This was replicated for posterior-lateral (L) PkT (R): $P \leq 0.001, r=0.42$; PkT (L): $P=0.002, r=0.39 ; \operatorname{AvF}(\mathrm{L}) P=0.003, r=0.38$ and AvT (L) $P=0.002, r=0.39$. No significant correlation coefficients were displayed for $\operatorname{AvF}(\mathrm{L}), \operatorname{AvT}(\mathrm{L})$ and $\Theta$ when compared to $(\mathrm{R})$ and posteriorlateral stability $(P>0.05)$. The opposite was identified for $(\mathrm{L})$ posterior-lateral stability, where no significant correlation coefficients were displayed for $\operatorname{AvF}(\mathrm{R})$, AvT (R) and $\Theta(P>0.05)$. PM stability displaying small significant correlations.

(R) and (L) Ant stability displayed no significant correlation coefficients against any eccentric hamstring strength metrics $(\mathrm{P}>0.05)$. See table 2 for a summary of stability parameters and eccentric hamstring strength metrics for elite academy footballers.

\author{
***insert table 2 here $* * *$
}


The aim of the present study was to investigate the relationship between lower limb dynamic stability and eccentric hamstring strength in elite academy footballers. Main findings from this body of work highlight significant correlations between eccentric hamstring strength and PL/PM stability. However, analysis of correlations between Ant stability and eccentric strength demonstrated no significant relationships. Significant correlations were identified for PL and PM stability with the magnitude ranging from small to moderate, with PkT and AvT displaying the highest range $(\mathrm{r}=0.36-0.62)$, compared to Force values $(r=0.31-0.39)$. This could potentially be explained by the relative nature of force output to body mass ${ }^{26}$. Break angle also displayed no significant correlations. This metric can provide an interpretation of muscle architecture, but does not relate to the actual strength output of the muscle. Observations of this metric were made to determine whether architecture of the muscle had any influence on directional stability performance, due to changing demands in the varying directions of movement tested within the YBT.

Previous work has often referred to dynamic stability, as proprioception ${ }^{11}$. Proprioception has consistently been highlighted as being, 'the body's ability to sense movements within joints and to have a knowledge of where these joints are in relation to space' ${ }^{31}$. This operational definition within an applied environment may cause some confusion, as measures, tests and exercises actually relate to an effected output and not the physiological proprioceptive process. To measure proprioception as a whole function is difficult and as practitioners in sport, we are more concerned about the effected output. Consideration has been given to this in the present study and identification of the relationship between eccentric hamstring strength and dynamic stability identified. The present study also considers the directional nature of dynamic stability in relation to functional strength, something not reflected in previous literature in this area ${ }^{12}$. Quantification of dynamic stability within research is consistently debated, with no gold standard functional test identified ${ }^{32}$. In the present study, the YBT was utilised to determine stability measures. ACL injuries are commonly associated with high load anterior shearing force, often accompanied with rotation ${ }^{16}$. Utilisation of the YBT provided an opportunity to analyse 
directional stability, allowing association to be made between the anatomical function of the hamstring and the stability score. Findings from the present study provide practitioners with an understanding of the contribution of eccentric hamstring strength on knee function, informing conditioning, rehabilitation and injury prevention protocols. However, small to moderate correlations support the acknowledgement throughout literature that hamstring and knee injuries are multi factorial and may not be the only consideration in injury risk reduction or rehabilitation protocols ${ }^{3,6}$.

\section{Previous research has focussed on quantifying proprioception/dynamic stability with an overall stability} score $^{21}$, when examining the influence of eccentric strength. This limits interpretation of the relationship between eccentric hamstring strength, knee function and its relevance to functional performance. In the present study relationships were established between eccentric hamstring strength parameters and directional stability (inclusive of; (R) and (L) Ant, PM and PL). Significant correlations were identified for (R) and (L) PM stability, noted between all strength parameters of PkT, AvT and AvF (L) and (R). Suggesting that increased eccentric hamstring strength in elite academy footballers, contributes to greater stabilisation in this plane of movement. During the performance of the YBT the hamstrings act as a dynamic stabiliser at the knee, reducing stress through the joint. The lunge position completed when performing the YBT consists of flexion of the knee and increased rotational stress through the joint. Consideration of the mechanism of injury associated with common knee injuries sustained in elite football, are associated with this movement pattern ${ }^{16}$. Potentially indicating that sports medicine practitioners should consider this in their choice of exercise prescription in the pursuit of decreasing injury risk.

During functional movement patterns performed during football specific movements anterior stabilisation is required to support key structures in the knee, such as the ACL. This movement pattern provides the most contentious debate in the present findings. Consideration must be given to the position of the athlete when performing this measure in the YBT, as the hamstring muscle is not contracting eccentrically at reach position. However, to control further stress on the knee and reverse the movement pattern the hamstrings will sustain eccentric load and if the functional strength of the athlete is not 
apparent, there is potential to perform poorly on the stability test. Current findings indicate strength has no influence on this. This potentially supports cardarvic research suggesting that reduced mechanoreceptors detected in the ACL, may result in a delayed proprioceptive response when performing functional tasks ${ }^{22}$. Previous research has identified the influence of decreased ankle dorsiflexion, poor antagonistic function, and hip mobility, to name a few ${ }^{33}$. These factors may explain poor anterior or overall stability output, however; only provide limited explanation to why relationships were found in other planes of movement. Consideration must also be given to limb dominance as this may demonstrate further relationships between eccentric strength and dynamic stability ${ }^{34}$. Future research should investigate these considerations in order to establish the multi-factorial nature of injury.

Dynamic stability is the effected output generated through proprioceptive function. During completion of this movement pattern, the knee and specifically the tibia are subjected to an anterior force, as it would during functional performance when decelerating in an anterior plane. Cardarvic research indicates that the ACL has fewer mechanoreceptors contained within it when compared to the surrounding structures within the joint ${ }^{11}$. Potentially, suggesting that there may be a delayed signal within the proprioceptive pathway to generate the required output through this plane of movement. This said other aetiological factors cannot be discounted such as antagonistic function, ankle dorsiflexion or hip mobility ${ }^{33}$.

It is well documented throughout literature that muscle architecture is key to reducing hamstring injury risk $^{24}$. Findings within the present study showed no significant relationships between any stability parameter and $\Theta$. An explanation of this through observation of potential differences in position and resultant muscle length when performing the two tests may be relevant. Consideration to the mechanism of injury for hamstring and ACL provides a potential explanation as to why no relationship was found in the current study. However, further research in this area would be required. Research indicates that the ACL is the most commonly injured ligament in the knee ${ }^{35}$, with increases in re-rupture and poor return of the athlete to the same level pre-injury ${ }^{6}$. Common mechanisms associated with ACL are linear motions from either a rapid acceleration or deceleration or excessive anterior force through the knee 
joint ${ }^{16}$. The findings in the current research highlight relationships between higher eccentric strength scores and lower dynamic stability scores, with the exception of AP stability and $\Theta$. Consideration therefore, that increased eccentric strength has a positive influence on stability of the knee is suggested. Further research in this area should focus its attention on intervention training protocols to examine their effect on key aetiological contributors to injury.

\section{Conclusion}

Eccentric hamstring strength metrics are positively associated with PM and PL directional stability performance during the YBT, with no relationships existing between strength and anterior stability.

Caution must be applied when interpreting the significance of the findings due to the small to moderate relationships identified. However, importantly interpretation of the findings suggest that despite increases in functional hamstring strength, a main stabiliser of the knee during functional performance, has no influence on the stability of the knee. Thus, implying that despite the intervention of eccentric hamstring strengthening protocols within elite footballers, players can potentially still be at an increased risk of sustaining ACL injury. Careful consideration must be given by key stakeholders within performance departments to quantification of injury risk parameters of strength and stability, interpretation of their results and application in conditioning and injury prevention protocols.

\section{Practical Implications}

- Relationships are evident between PM and PL directional stability and eccentric strength metrics, which potentially indicates the need for practitioners to consider the inclusion of eccentric strengthening programmes. However, further research is required in the format of a training study to identify cause-effect relationships.

- Although relationships were identified between eccentric strength and PM/PL directional stability, none were identified with AP stability. Sports medicine practitioners cannot disregard other aetiological factors associated with poor stability performance and must not discount these in injury risk reduction protocols. 
- Increases in eccentric hamstring strength parameters may reduce muscle injury risk, but effect on joint injury risk is not conclusive.

\section{References}

1. Ekstrand J, Hagglund M, Walden M. Injury Incidence and Injury Patterns in Professional Football: The UEFA Injury Study. Br J Sports Med 2011; 45(7):553-558.

2. Woods C, Hawkins RD, Maltby S. The Football Association Medical Research Programme: an audit of injuries in professional football. Analysis of Hamstring Injuries. Br J Sports Med 2004; 38(1):36-41.

3. Ekstrand J, Walden M, Hagglund M. Hamstring Injuries Have Increased by $4 \%$ Annually in Men's Professional Football, Since 2001: A 13 Year Longitudinal Analysis on the UEFA Elite Club Injury Study. Br J Sports Med 2016; 50(12):731-8.

4. Erickson BJ, Harris JD, Cvetanovich GL, et al. Performance and return to sport after anterior cruciate ligament reconstruction in male Major League Soccer players. Ort J Sports Med; 2013; 11(1): 553-558.

5. Krutsch W, Zeman F, Zellner J, et al. Increase in ACL and PCL injuries after implementation of a new professional football league. Knee Surg Sports Traumatol Arthrosc 2016; 24(7): 2271 2279.

6. Walden M, Hagglund M, Magnusson H, et al. ACL Injuries in Men's Professional Football: A 15-Year Prospective Study on Time Trends and Return-to-Play Rates Reveals only 65\% of Players Still Play at the Top Level 3 Years after ACL Rupture. Br J Sports Med 2016; 50(12):17.

7. Messer D, Bourne M, Timmins R, et al. Eccentric knee flexor strength and hamstring injury risk in athletes with history of anterior cruciate ligament reconstruction. $\mathrm{Br} \mathrm{J}$ Sports Med 2017;51:284-413.

8. Barengo NC, Meneses-Echavez JF, Robinson RV, et al. The Impact of the FIFA 11+ Training Program on Injury Prevention in Football players: A Systematic Review. Int J Environ Pub Health 2014; 11(11):11986-12000 
9. Rhodes D, McNaughton L, Greig M. The Temporal Pattern of Recovery in Eccentric Hamstring Strength Post Soccer Specific Fatigue. Res Sports Med 2018; 10(8):1-12.

10. Proske U and Morgan DL. Muscle Damage from Eccentric Exercise: Mechanism, Mechanical Signs, Adaptation and Clinical Applications. J Physiol 2001; 537(pt2):333-345.

11. Changella PK, Selvamani K, Ramaprabhu. A Study to Evaluate the Effect of Fatigue on Knee Joint Proprioception and Balance in Healthy Individuals. Int J Sci Res Pub 2012; 2(3):18511857.

12. Booysen MJ, Gradidge PJ, Watson E. The relationships of eccentric strength and power with dynamic balance in male footballers. J Sports Sci 2015; 33(20): 2157-2165.

13. Hyun-Jung K, Jin-Hyuck L, Sung-Eun A, et al. Influence of Anterior Cruciate Ligament Tear on Thigh Muscle Strength and Hamstring-to-Quadricep Ratio: A Meta Analysis. PLoS One 2016; 11(1):1-11.

14. Arnason SM, Birnir B, Gudmundsson G, et al. Medial Hamstring Muscle Activation Patterns are Affected 1-6 Years after ACL Reconstruction Using Hamstring Autograft. Knee Surg Sports Traumatol Arthrosc 2014; 22(5):1024-1029.

15. Kim HJ, Lee J H, Ahn SE, et al. Influence of Anterior Cruciate Ligament Tear on Thigh Muscle Strength and Hamstring-to-Quadriceps Ratio: A Meta Analysis PLoS One 2016; 11(1):1-11.

16. Alentorn-Geli E, Myer GD, Silvers HJ. Prevention of Non-Contact Anterior Cruciate Ligament Injuries in Soccer Players. Part 1: Mechanisms of Injury and Underlying Risk Factors. Knee Surg Sport Traumatol Arthrosc 2009; 17(7): 705-729.

17. Harput G, Kilinic E, Ozer H, et al. Quadriceps and Hamstring Strength Recovery During Early Neuromuscular Rehabilitation After ACL Hamstring-Tendon Autograft Reconstruction. J Sports Rehab 2015; 24(4):398-404.

18. Besier TF, Lloyd DG, Cochrane JL. External loading of the knee joint during running and cutting manoeuvres. Med Sci Sports Ex 2001; 33(7):1168-1175.

19. Hewett TE, Di Stassi SL, Myer GD. Current Concepts for Injury Prevention in Athletes after Anterior Cruciate Ligament Reconstruction. Am J Sports Med 2013; 41(1):216-224. 
20. Bryant AL, Clark RA, Pua YH. Morphology of Hamstring Torque-Time Curves following ACL Injury and Reconstruction: Mechanisms and Implications. J Orthop Res 2011; 29(6):907-914.

21. Melnyk M and Gollhofer A. Submaximal Fatigue of the Hamstrings Impairs Specific Reflex Components and Knee Stability. Knee Surg Sports Traumatol Arthrosc 2007; 15(5):525-532. Proprioception and Postural Stability. PLoS One 2015; 10(9):1-10.

23. Petersen J, Thorborg K, Nielsen BM, et al. Preventive effect of Eccentric Training on Acute Hamstring Injuries in Men's Soccer: A Cluster-Randomized Control Trial. Am J Sports Med 2011; 39(11):2296-2303.

24. Opar DA, Piatkowski T, Williams MD, et al. A novel device using the Nordic hamstring 398 exercise to assess eccentric knee flexor strength: a reliability and retrospective injury study. $\mathbf{J}$ Orthop Sports Phys Ther 2013; 43(9):636-640.

25. Hinman M. Factors Affecting Reliability of the Biodex Balance System: A summary of Four Studies. J Sport Rehabil 2000; 9(3):240-252.

26. Buchheit M, Cholley Y, Nagel M, et al. The effect of body mass on eccentric knee-flexor strength assessed with an instrumented Nordic hamstring device (NordBord) in football players. Int J Sports Physiol Perform 2016; 11(6):721-726.

27. Sedliak M, Haverinen M, Hakkinen K. Muscle Strength, Resting Muscle Tone and EMG Activation in Untrained Men: Interaction Effect of time of Day and Test Order-Related Confounding Factors. J Sports Med Phys Fit 2011; 51:560 - 570.

28. Lee JWY, Mok K, Chan HCK, et al. Eccentric hamstring strength deficit and poor hamstringto-quadriceps ratio are risk factors for hamstring strain injury in football: A prospective study of 146 professional players. J Sci Med Sport 2017; 21(8):789-793.

29. Smith LJ, Creps JR, Bean R, et al. Performance and Reliability of the Y Balance Test in High School Athletes. J Sports Med Phys Fit 2017; 58(11):1671-1675.

30. Robinson R. and Gribble P. Kinematic predictors of performance on the star excursion balance test. J Sport Rehab 2008; 17(4):347-357. 
31. Han J, Waddington G, Adams R, et al. Assessing Proprioception: A Critical Review of Methods. J Sport Health Sci 2015; 5(1):80-90.

32. Dawson N, Dzurino D, Karleskint M, et al. Examining the reliability, correlation and validity of commonly used assessment tools to measure balance. Health Sci Rep 2018; 1(12): 1-8.

33. Kang MH, Kim GM, Kwon OY, et al. Relationship between the kinematics of the trunk and lower extremity and performance on the Y-balance test. PMR 2015; 7(11): 1152-1158.

34. Brophy R, Silvers H J, Gonzales T, et al. Gender Influences: The Role of Leg Dominance in ACL Injury Among Soccer Players. Br J Sports Med 2010; 44(10): 69469.

35. Silvers-Granelli HJ, Bizzini M, Arundale A, et al. Does the FIFA 11+ injury prevention programme reduce the incidence of ACL injury in male soccer players? Clin Orth Rel Res 2017; 475(10):2447-2455.

\section{Tables Legends}

Table 1: Displaying Mean and Standard Deviation.

Table 2: Displaying Relationships between Stability Parameters and Eccentric Hamstring Strength Metrics for Elite Academy Footballers.

\section{Figure Legends}

Figure 1: Image of the standardisation of the camera used in order to determine the break point angle of the NHE. 